\title{
A 'Universal' Bottom-up Sol-gel Based Synthesis of Pzt (1-x)/x Submicrometric Structures: 1. Dynamic Light Scattering Characterization of Precursor Stability and Aggregation Kinetics
}

\author{
Amaury Suárez-Gómez z,b*, Vladimir Rodríguez-Iglesias ${ }^{c}$ \\ ${ }^{a}$ Centro de Ciencias Aplicadas y Desarrollo Tecnológico, Universidad Nacional Autónoma de \\ México - UNAM, Cd. Universitaria, Circuito Exterior, CP 04510, Coyoacán, Distrito Federal, México \\ ${ }^{b}$ Centro Universitario de los Valles, Universidad de Guadalajara-UdG, Carr. Guadalajara-Ameca \\ Km 45.5, CP 46600, Ameca, Jalisco, México \\ ${ }^{c}$ Facultad de Ingeniería, Universidad Autónoma del Carmen - UNACAR, Campus III, Avenida Central \\ s/n, Esq. con Fracc. Mundo Maya, CP 24115, Ciudad del Carmen, Campeche, México
}

Received: May 6, 2015; Revised: May 25, 2015

\begin{abstract}
This work proposes an extension of a sol-gel based synthesis route for obtaining highly hydrophobic, uniformly nanosized and stable precursor solutions of lead zirconate titanate $\left[\mathrm{Pb}\left(\mathrm{Zr}_{1-\mathrm{x}} \mathrm{Ti}_{\mathrm{x}}\right) \mathrm{O}_{3}\right.$ or PZT $(1-\mathrm{x}) / \mathrm{x}]$ for any given $(1-\mathrm{x}) / \mathrm{x}$ Zr:Ti ratio. Several representative PZT compositions were chosen and their precursors were studied by taking into account the time dependence of the mean particles size andthe predominant aggregation kinetics. Size distributions were recorded by the Dynamic Light Scattering technique and the aggregation kinetics was explored by considering a Diffusion-Limited Colloidal Aggregation model. Transmission electron microscopy was also used to characterize some morphological features of our resultant precursor nanoparticles. Our final results supports the universalization of this approach to any PZT composition and its extension to other material systems. This work is intended to be the first on a series dealing with the sol-gel based synthesis of any PZT material at any scale using a bottom-up approach.
\end{abstract}

Keywords: PZT, nanoparticles, sol-gel, dynamic light scattering, aggregation kinetics

\section{Introduction}

In recent years, there has been an especially devoted interest in obtaining nano- and submicron- powders not only for synthesizing nanoceramic materials in bulk form but also to allow a cheaper and faster technological escalade by providing a reasonable compatibility and integration with current standard industry procedures as well as a better chance for miniaturization in the nano-, meso- and submicrometric working scales ${ }^{1}$. The appearance of an amazing amount of size related effects in most of the inorganic bulk materials when average grain size was below a certain limit, commonly around $1 \mu \mathrm{m}$, triggered a lot of research in this field and the traditional solid statepowder-based routes of synthesis were modified by using purer starting reagents, developing new sintering and milling techniques while performing longer millings, etcetera. However, interfacial contamination, local inhomogeneities and morphological qualities were, and still are, recurrent problems intimately related to the powderbased physical methods.

Chemical synthesis routes, however, practically eliminate the interfacial and inhomogeneity issues while consequently paving the way to the crystallization of a material with high morphological quality. It must be mentioned that the most relevant characteristic of any chemical route -the mixing of the appropriate organic precursors at molecular level- is also an important factor when considering the relatively lower

*e-mail: amaury.sg@gmail.com synthesis temperatures commonly required for attaining a good crystallization of the desired material. Among the most widely used chemical routes, the sol-gel based ones have proved quite successful for inorganic materials synthesis since they were first introduced in the late 70's-early 80's for obtaining thin and/or thick films that were later integrated in more complex and robust electronic systems ${ }^{2}$. On the other hand, the high versatility of the sol-gel method is usually limited by the availability and the chemical nature of the starting reactants and this fact, along with the particularly targeted branch of application, has implied a lot of discussion and research for more than 30 years now ${ }^{3}$.

The sol-gel based chemical routes are also one of the best candidates since the mid 90 's for synthesizing nanosystems in a myriad of ways: nanotubes, nanorods, nanofibers, electrodeposited templated nanostructures, nanofilms, nanoparticles, and so on, giving rise to fundamental nanoscience studies and applications at a relatively low $\operatorname{cost}^{4,5}$. However, research in the field of nanopowders or nanoceramics synthesized by means of sol-gel routes was not significant until almost a decade later $^{2,6-8}$; the year 2009, for instance, saw a remarkable increment in the number of publications where the synthesis of this kind of materials was investigated using sol-gel based chemical routes. Along with this fact, sol-gel synthesis provides the very same benefits that have been employed in building nanosystems from the bottom-up, as famously 
coined Richard Feynman ${ }^{9}$, thus allowing its use as a tool for effectively build the nano-, the meso- and, in some cases, the submicron- final volumetric material using that very same approach. Even though the synthesis of bulk nanoceramics implies some post-processing heat treatments that somehow tend to 'destroy' the nanostructured building blocks, which are organic by nature, there is a huge amount of research that needs to be done in order to tune, both physically and chemically, the whole synthesis process to obtain a highly crystalline material, with a minimum waste of energy, with controlled porosity and morphologically uniform ${ }^{1,2,8,10,11}$. This kind of study, specifically focused on a bottom-up approach for the sol-gel based synthesis of nanoceramics, just started to grow in the beginning of this decade and still can be considered an emerging field in the area.

\subsection{Background}

In the last few years, we have been focused on this kind of approach for the synthesis of lead zirconate titanate [PZT; with general formula $\left.\mathrm{Pb}\left(\mathrm{Zr}_{1-\mathrm{x}} \mathrm{Ti}_{\mathrm{x}}\right) \mathrm{O}_{3}\right]^{12-14}$, a well-known $\mathrm{ABO}_{3}$ ferroelectric perovskite with a wide range of industrial applications since the early 50's of the last century ${ }^{15}$ and, still nowadays, a technologically relevant material often considered the backbone of the piezoceramics industry and with numerous applications that range from sensors, actuators and accelerometers to ultrasonic equipment, MEMS, imaging devices and microphones, among others.

As for the chosen synthesis route, we use an acetate/ alkoxides sol-gel three-steps route modified with acetylacetone as chelating agent and stabilized with 2-methoxyethanol (2-MOE) that has been clearly detailed previously ${ }^{13}$ and later expanded and improved to take into account several important physicochemical factors ${ }^{14}$. One of those relevant factors under study has been the sol stability with time -or shelf life, as is usually termed- and the description of the aggregation kinetics ${ }^{12}$ in order to achieve homogeneous solutions for any future processing: nanopowder synthesis, chemical solution deposition, electrophoretic deposition, simple wetting, and others.

Due to the crucial role of sol-gel stability on the aforementioned processing steps, it is very important to keep control over some parameters that affect the solution stability and, most of all, the average particles size. Particularly, particles size can be an indicator of some undesirable processes that could be takingplace in the sol: aggregation, flocculation and sedimentation; aggregation, even thoughis a reversible process, is a good indicator of instability sincethe other two processes, whichare not reversible, generally follows after some time.

In this respect, the aging time dependences of some physical parameters are intimately correlated and heavily determinant on the stability of colloidal dispersions; for instance, the $\mathrm{pH}$ of the solution is a key factor for fixing the thickness of the ionic layers surrounding any givencharged colloidal particle (Stern layers) while affecting, at the same time, the Zeta potential,the electrophoretic mobility and the aggregation mechanisms as well as the apparenthydrodynamic particle size. As a consequence, the average particles size is the final result ofthe conjugate action of all the physicochemical variables hardly mentioned before. It is alsothe definitive experimental variable on which any post processing technique should bebased on as it explicitly defines the size range of the so called "building blocks" for bottom-upstudies or applications ${ }^{11}$.

According to our previous reports on this particular topic, we have obtained several results that must be highlighted due to their relative importance from a technological point of view:

a) Using propoxides as starting reagents leads to a better crystallization than when using butoxides or any other alkoxide with a larger alkyl chain,

b) The chelation reaction should be carried out avoiding any further byproduct,

c) A final PZT precursor concentration of $0.35 \mathrm{M}$ in 2-MOE is considered optimal for sample stability and proper characterization,

d) Nanoparticles in the sol are positively charged; $\mathrm{pH}$ is reasonably well below the isoelectric point for the system,

e) After several days of aging, the variations of the average particle size are independent of the variations of $\mathrm{pH}$,

f) When optimizing the chelation reaction as suggested in b), the fractal dimension of the precursor solution have slight variations during the aging process.

Even though some of these results are going to be mentioned and briefly explained later on the text, it should be pointed out that they were obtained exclusively for the sol-gel based PZT 53/47 precursor. Therefore, this work and some others that will follow shortly, will try to 'universalize' our study by extending its scope and applicability for any chosen Zr:Ti molar ratio.

On this paper, we will start by focusing on the aging stability and the aggregation kinetics of sol-gel based PZT (1-x)/x precursor solutions and, accordingly, several distinctive $\mathrm{Zr} / \mathrm{Ti}$ ratios were chosen for this study; PZT-25/75 (tetragonal ferroelectric phase, $F_{T}$ ), PZT-53/47 (high temperature rhombohedral ferroelectric phase on the morphotropic phase boundary, $F_{R}{ }^{(H T)}$ ), PZT-60/40 (high temperature rhombohedral ferroelectric phase, $\left.F_{R}{ }^{(H T)}\right)$, PZT-80/20 (low-temperature rhombohedral ferroelectric phase, $F_{R}^{(L T)}$ ) and PZT-95/05 (orthorhombic antiferroelectric phase, $A_{O}$ ). Our study will be solely based on dynamic light scattering (DLS) measurements and on conventional and high-resolution transmission electronic microscopies (TEM and HR-TEM, respectively) micrographs.Currently, there are no known extensive reports in the literature directly concerned to thestudy of the time dependence of the average particles size in aPZT precursor sol.

\section{Experimental}

\subsection{Sol-gel synthesis}

Starting reagents were lead (II) acetate trihydrate ( $\mathrm{Pb}(\mathrm{OAc}) 2 \cdot 3 \mathrm{H} 2 \mathrm{O}$, Aldrich, 99.99\% pure), acetic acid (HOAc, Sigma-Aldrich, 99.7\%), acetylacetone 
(AcacH, Sigma Aldrich, 99\%), 2-methoxyethanol anhydrous (2-MOE, Sigma-Aldrich, 99.8\%), zirconium(IV) propoxide ( $\mathrm{Zr}(\mathrm{OPr}) 4$, Aldrich, $70 \mathrm{wt} \%$ in 1-propanol), and titanium(IV) propoxide (Ti(OiPr)4, Aldrich, 98\%) that were mixed according to the three steps reaction shown in Figure 1.

First, lead acetate is dissolved in acetic acid with a 1:3 molar ratio at $120{ }^{\circ} \mathrm{C}$ for 3 hours in order to form solution A and, in a second step, a solution B is obtained when stoichiometric amounts of zirconium and titanium propoxides were mixed with acetylacetone at the optimal chelation ratio $\left(R_{\text {OPTIMAL }}\right)$ that, as shown in an earlier work ${ }^{16}$, is dependent on the chosen $\mathrm{Zr} / \mathrm{Ti}$ ratio and is ideally given by $R_{\text {optimal }}(\mathrm{x})=2(2-\mathrm{x})$ according to the reaction:

$$
\begin{aligned}
& (1-\mathrm{x}) \operatorname{Zr}\left(O^{\mathrm{i}} \mathrm{Pr}\right)_{4}+\mathrm{x} T i\left(O^{\mathrm{i}} \operatorname{Pr}\right)_{4}+2(2-\mathrm{x}) A c a c H \frac{\mathrm{T} \sim 90^{\circ} \mathrm{C}}{\mathrm{t} \sim 4 h r s} \ldots \\
& \ldots 2(2-\mathrm{x}) H O^{\mathrm{i}} \mathrm{Pr}+(1-\mathrm{x}) \operatorname{Zr}(A c a c)_{4}+\mathrm{x} T i(A c a c)_{2}\left(O^{\mathrm{i}} \mathrm{Pr}\right)_{2}
\end{aligned}
$$

In the final step of the synthesis, solutions A and B are mixed in the presence of 2-MOE until the concentration of $0.35 \mathrm{M}(5.0<\mathrm{pH}<5.8)$ is reached. In this new chemical scenario, the dissolved lead acetate complex can react with 2-MOE forming a very stable acetate-methoxyethoxylead complex ${ }^{17}$ that, along with the chelated metal complexes alreadyformed, may lead to turn this final solution into a hydrophobic sol, poorly hydrolysable and, therefore, very stable.

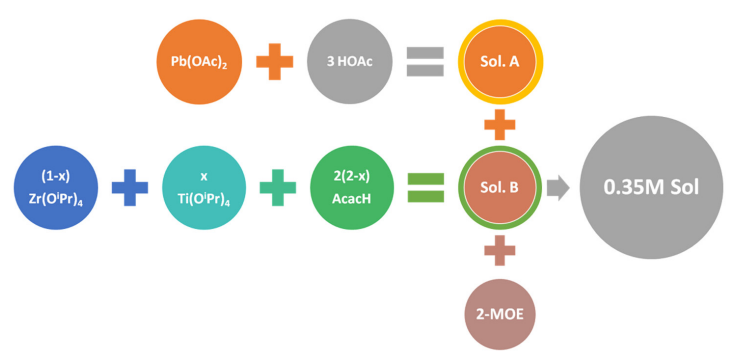

Figure 1. Schematic of the sol-gel based route used in this work.

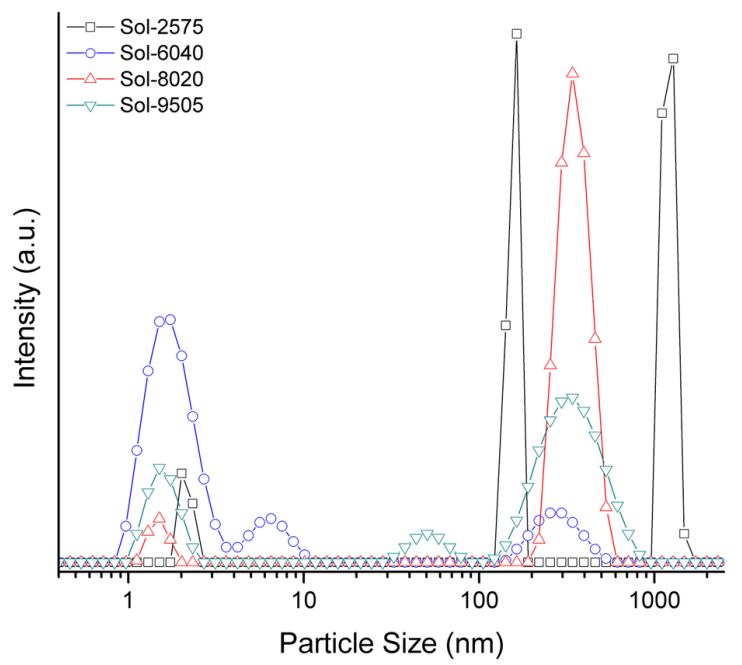

(a)
For the sake of clarity, every precursor solution will be referred hereafter as the plain combination of the zirconium and titanium molar fraction preceded by 'Sol-', i.e. Sol-6040 refers to the synthesized precursor of the PZT $60 / 40$ composition.

\subsection{Experimental techniques}

After stirring for about 10 hours, approximately $1.5 \mathrm{ml}$ of as-synthesized sols were stored in polystyrene cells and particles size measurements were carried out by means of the dynamic light scattering (DLS) technique in a Zetasizer Nano ZS90 (Malvern Instruments Ltd.) equipped with a HeNe laser source. Size distributions curves were recorded for different aging times, ranging from early days after synthesis up until $\sim 35$ days. This period of time has proven critical for the sol-gel precursor as dramatic $\mathrm{pH}$ variations beyond this aging time are no longer registered up until 4 months of stocking reachinga quite noticeable stability around 5.5 $5^{12,14}$. Samples were stocked and characterized at an average room temperature of $\sim 15^{\circ} \mathrm{C}$, only conditioned by the time of the year when these measurements were made.

Our samples were also characterized by the conventional and high resolution Transmission Electron Microscopytechniques, TEM and HR-TEM. In both cases, a few drops of the synthesized sols were deposited on coppergrids, evaporated afterwards and put under a JEOL 2010 (TEM) and a JEOL JEM2200 (HR-TEM) microscope with Omega filterand a spherical aberration corrector.

\section{Results and Discussion}

As has been reported before for different experiments ${ }^{12,16}$, there is a noticeable change in the size distribution curves as all our samples aged. At first, Figure 2a, as-prepared sols featured multimodal distributions with a noticeable percentage of particles that could even have sizes sometimes above $1 \mu \mathrm{m}$. As solutions are aged, Figure $2 b$, these distributions tend to be unimodal featuring a mean particle size well below $10 \mathrm{~nm}$. Consequently, it is strongly suggested to use aged samples for any post-processing step at any projected working scale.

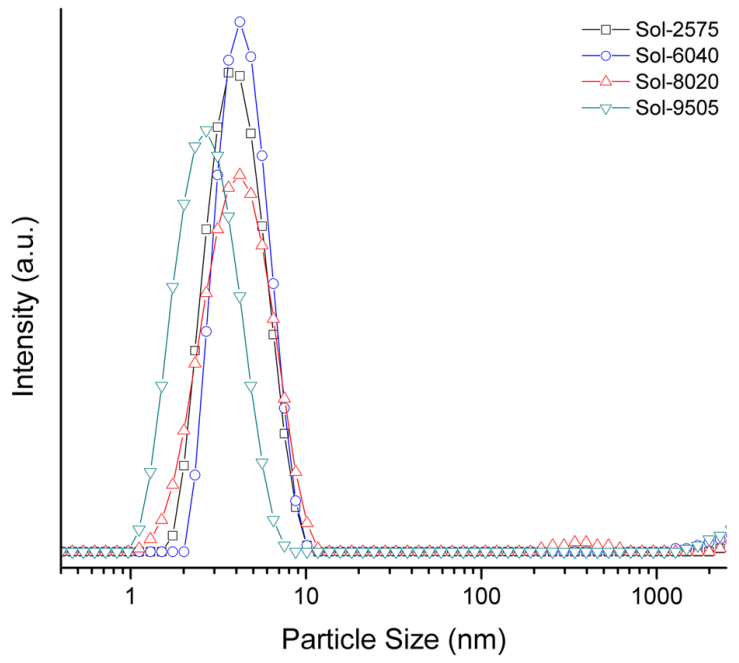

(b)

Figure 2. Size distribution curves as recorded by dynamic light scattering for samples aged for (a) 1 day and (b) 25 days after synthesis. 
We have also found that there is a correlation between the chelation ratio, $R$, and the aging time at which our samples become mostly unimodal -referred by us as 'unimodal age'- ${ }^{16}$ being $\sim 20-25$ days when working with $R=R_{\text {OPTIMAL }}$ and this is also shown in Figure $2 \mathrm{~b}$. This behavior is explained on the basis of the complete chelation proposed in Equation 1 by making the plausible assumption that solution $\mathrm{B}$ is a dispersion containing the $\mathrm{Ti}(\mathrm{Acac})_{2}(\mathrm{OPr})_{2}$ compound at molecular level and submicrometric aggregates of $\mathrm{Zr}(\mathrm{Acac})_{4}$ that are redissolved afterwards when mixed with the acetate rich solution A and 2-MOE.

Results of the recorded average particle size $(<d>)$ vs. aging time are depicted in Figure 3. Due to the fact that multimodal distributions exist during the first days after synthesis, it is worth to mention that we considered, when graphing these results, the average particle size of the leftmost population distribution.

As is also shown, non-linear least squares fitting (NLLSF) were carried out based on a diffusion-limited colloid aggregation (DLCA) regime of aggregation kinetics ${ }^{18}$ as described by the relationship:

$<d>=d_{0}+A t^{1 /<d_{f}>}$

where the parameters $A, d_{0}$ and $\left\langle d_{F}>\right.$ were fitted to the available experimental data. According to our chemical route and experimental procedure, a reaction-limited colloid aggregation (RLCA) ${ }^{19}$ scenario was discarded because any reaction-limited process should occur within a few hours after synthesis and that is why samples were stirred for almost 10 hours right afterwards.

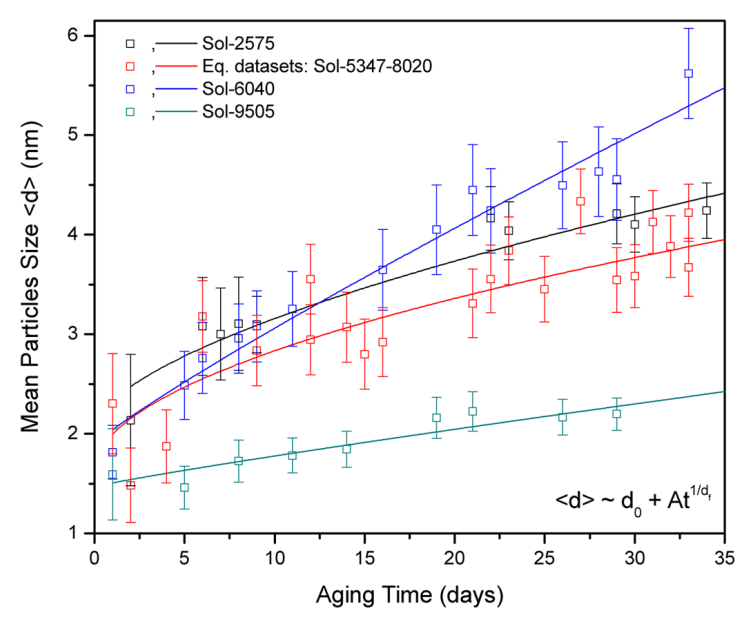

Figure 3. Evolution of the mean particles size as samples aged considering only the statistically independent datasets. Fittings are based on a DLCA aggregation mechanism.

Table 1. Summary of the paired t-tests carried out to our whole datasets. Statistically equivalent groups ( $\mathrm{p}$-value $>0.05$ ) are highlighted.

\begin{tabular}{lcccc}
\hline & Sol-2575 & $\begin{array}{c}\text { Eq. datasets } \\
\text { Sol-5347-8020 }\end{array}$ & Sol-6040 & Sol-9505 \\
\hline $\boldsymbol{d}_{\boldsymbol{0}}$ & $2.1 \pm 0.3$ & $1.8 \pm 0.4$ & $1.9 \pm 0.3$ & $1.6 \pm 0.1$ \\
$\boldsymbol{A}$ & $0.3 \pm 0.1$ & $0.3 \pm 0.1$ & $0.15 \pm 0.09$ & $0.014 \pm 0.007$ \\
$\boldsymbol{d}_{\boldsymbol{f}}$ & $1.6 \pm 0.2$ & $1.7 \pm 0.3$ & $1.1 \pm 0.2$ & $0.8 \pm 0.1$ \\
\hline
\end{tabular}

In order to explore the significance of the increasing trend that can be seen in Figure 3, we first carried out Kolmogorov-Smirnov ${ }^{20}$ normality tests that were followed by a statistical analysis based on two-tailed paired t-tests to our datasets. The results are shown in Table 1.

According to the usually accepted 0.05 tolerance criterion for the p-values obtained in these tests, samples Sol-5347 and Sol-8020 are not significantly different and, statistically speaking, should be considered as a unique dataset. This new fact is taken into account when showing the experimental results and NLLSF curves in Figure 3 and also when obtaining the fitting parameters that appear in Table 2.

According to these results, there is a fairly wide region of $\mathrm{Zr} / \mathrm{Ti}$ compositions where the average particles size can be estimated to a certain degree, especially after the 'unimodal age' is reached. As can be seen, for aging times longer than $\sim 22$ days we will have $3 \mathrm{~nm}<<d><6 \mathrm{~nm}$ for almost every composition under study as well as remarkable unimodal distributions.

However, in the case of the Sol-9505 precursor, we recorded a different behavior. For this $\mathrm{Zr}$-rich composition, the smaller values in the $\langle d>$ vs. $t$ dependence could be the evidence of a faster dilution of the $\operatorname{Zr}(\text { Acac })_{4}$ chelated species when the more reactive $\mathrm{Ti}(\mathrm{OPr})_{2}(\mathrm{Acac})_{2}$ compound is at a very small concentration. Figure 4 depicts the recorded size distribution curves for samples of about 10 days old and a clear difference can be established among the Sol-9505 precursor and the others.

As can be expected, the rightmost populations tend to decrease with the $\mathrm{Zr}$ concentration even though their average values tend to be higher; however, for the $\mathrm{Zr}$-richest solution the rightmost population is almost absent. The probable existence of a limiting $\mathrm{Zr} / \mathrm{Ti}$ ratio between 80/20 and 95/05 for which this behavior starts to be preponderant is something that must be studied but it could be hypothesized from our current discussion. This could also be the proposal for further studying the dilution competition between the chelated species when in the presence of 2-MOE right after the final step of the synthesis.

The more relevant fitting parameter shown in Table 2 is the exponent $d_{F}$ which corresponds to the average fractal dimension of the colloidal aggregates. It should be considered that those $d_{F}$ values were calculated by taking into account only the average size measured for the leftmost distribution curves and that, especially before the 'unimodal age' is reached, the coexistence of two or more populations has been neglected. This fact could lead us to reconsider the physical meaning of the calculated value for $d_{F}$ as exclusively pertaining to a single size interval and not to the whole colloidal dispersion. However, it works as a good estimator for the fractal dimension of the building blocks for future PZT-based nano- or submicron- materials.

In this case, $d_{F}$ values $\left(0.75<d_{F}<1.75\right)$ are all around 1 and 2, which is an expected region for this kind of as-synthesized colloidal systems. However, the dimensional difference between the extreme values reinforces again our previous discussion regarding the somehow peculiar aggregation kinetics for the Zr-rich Sol-9505 precursor solution. It is obvious that an appropriate description on this respect requires a more accurate statistical model as well 
Table 2. Fitting parameters for the NLLSF made to our datasets based on a DLCA aggregation model.

\begin{tabular}{ccccccccccc}
\hline & \multicolumn{2}{c}{ Sol-2575 } & \multicolumn{2}{c}{ Sol-5347 } & \multicolumn{2}{c}{ Sol-6040 } & \multicolumn{2}{c}{ Sol-8020 } & \multicolumn{2}{c}{ Sol-9505 } \\
\cline { 2 - 16 } & t Stat. & Prob $>|\mathbf{t}|$ & t Stat. & Prob $>|\mathbf{t}|$ & t Stat. & Prob $>|\mathbf{t}|$ & t Stat. & Prob $>|\mathbf{t}|$ & t Stat. & Prob $>|\mathbf{t}|$ \\
\hline Sol-2575 & - & - & 6.42 & $8.40 \mathrm{E}-07$ & -2.70 & 0.01192 & 5.34 & $1.37 \mathrm{E}-05$ & 20.63 & $1.22 \mathrm{E}-17$ \\
Sol-5347 & 6.42 & $8.40 \mathrm{E}-07$ & - & - & 4.57 & $1.05 \mathrm{E}-04$ & 0.06 & 0.95014 & 40.80 & $4.19 \mathrm{E}-25$ \\
Sol-6040 & -2.70 & 0.01192 & 4.57 & $1.05 \mathrm{E}-04$ & - & - & 7.81 & $2.74 \mathrm{E}-08$ & 12.14 & $3.27 \mathrm{E}-12$ \\
Sol-8020 & 5.34 & $1.37 \mathrm{E}-05$ & 0.06 & 0.95014 & 7.81 & $2.74 \mathrm{E}-08$ & - & - & 9.36 & $8.17 \mathrm{E}-10$ \\
Sol-9505 & 20.63 & $1.22 \mathrm{E}-17$ & 40.80 & $4.19 \mathrm{E}-25$ & 12.14 & $3.27 \mathrm{E}-12$ & 9.36 & $8.17 \mathrm{E}-10$ & - & - \\
\hline
\end{tabular}

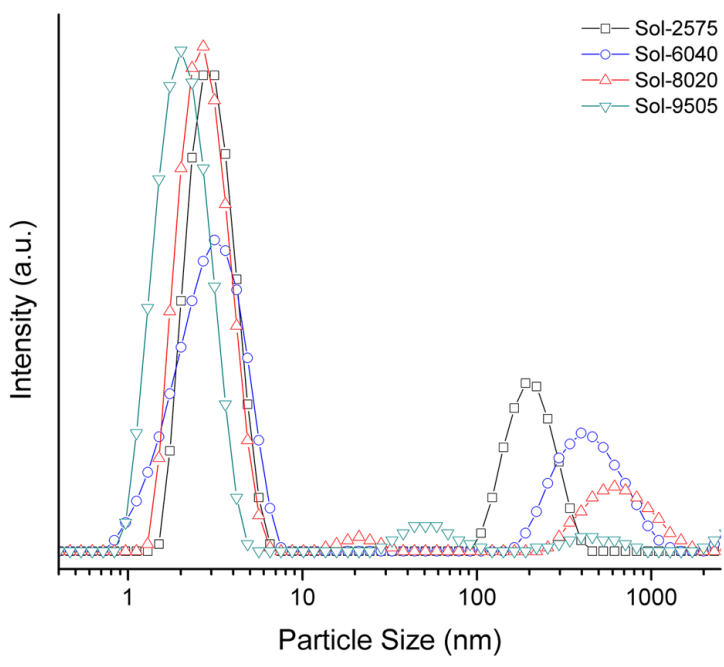

Figure 4. Size distribution curves as recorded by dynamic light scattering for samples aged for 10 days after synthesis.

as a thorough comprehension of the different competing processes taking place during the solution aging.

Another view of the aging behavior of our samples can be obtained by plotting the normalized aggregation velocities, $(d<d>/ d t)_{\text {NORMALIZED }}$, as in Figure 5. On this figure, the insets correspond to what was shown above in Figure $2 \mathrm{a}, \mathrm{b}$ and in Figure 4.

In this case, a more qualitative description of the aggregation process can be established according to what has been discussed before. For our specific synthesis methodology and environment, there is still a noticeable population in the $1 \mu \mathrm{m}$ range right after synthesis that diminishes to a great extent after just a few days. However, an almost complete extinction of these bigger particles is not recorded until more than 20 days of stocking have passed. From this moment on, precursor solutions show in all cases a remarkable unimodal distribution with average particle size below $10 \mathrm{~nm}$ for a few months. This fact may lead us to consider that fractal aggregates in these solutions tend to behave like Brownian particles with a small probability for gelification while, on average, aggregating according to diffusional mechanisms.

As described earlier in this work, our samples were stocked and measured at a room temperature of about $15^{\circ} \mathrm{C}$. To explore the influence of the stocking temperature, the same specimens for the PZT 53/47 precursor were synthesized and characterized, this time at a room temperature of about

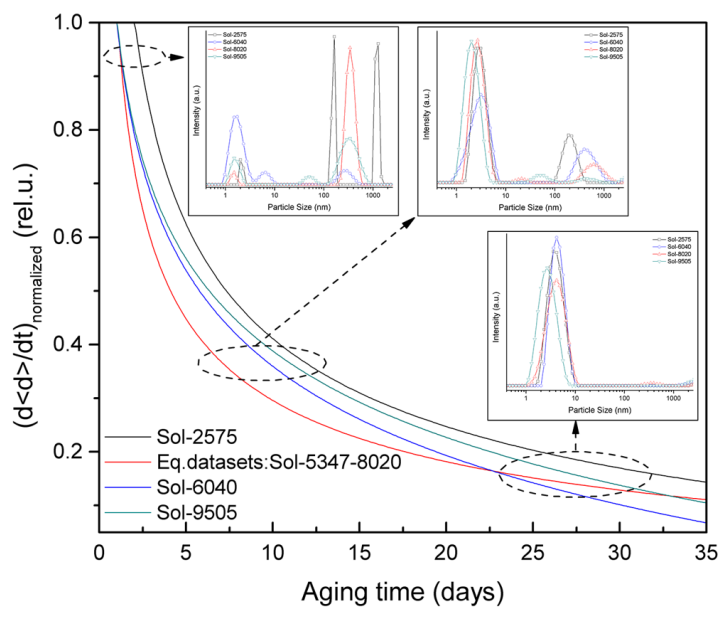

Figure 5. Another view of the aggregation kinetics according to the time evolution of the normalized aggregation velocities. The insets feature typical distribution behaviors that were mentioned and described earlier in this work.

$20^{\circ} \mathrm{C}$, and those results are shown in Figure 6 along with those of its homologue that were previously discussed.

Even though these new measurements fit very well into our previous discussions regarding stability and most probable aggregation mechanism, it is clearly observed a faster aggregation process when samples were stocked at higher temperatures as well as a smaller average fractal dimension $\sim 0.65$. Although this seems to be an expected result, due to the higher prominence of Brownian motion when temperature is raised, it also shows how the stocking environmental conditions constitute a very sensitive factor that should be taken into account for the development of any product directly related to this kind of precursor.

As stated above, aged samples were also characterized by the conventional as well as high resolution Transmission Electron Microscopy techniques -TEM and HR-TEM- and some of the obtained images are shown below. Figure 7 shows some typical views of our samples under different magnifications.

As can be seen, there has been some aggregation of the nanoparticles due to coalescence when solvent evaporates. However, a further exploration shows us the apparent coexistence of two different populations that are seen in more detail in Figure 8 and Figure 9.

In the first case, one of those aggregates shows multiple crystallographic orientations as an indication of being composed of several smaller nanoparticles and, as highlighted by the 


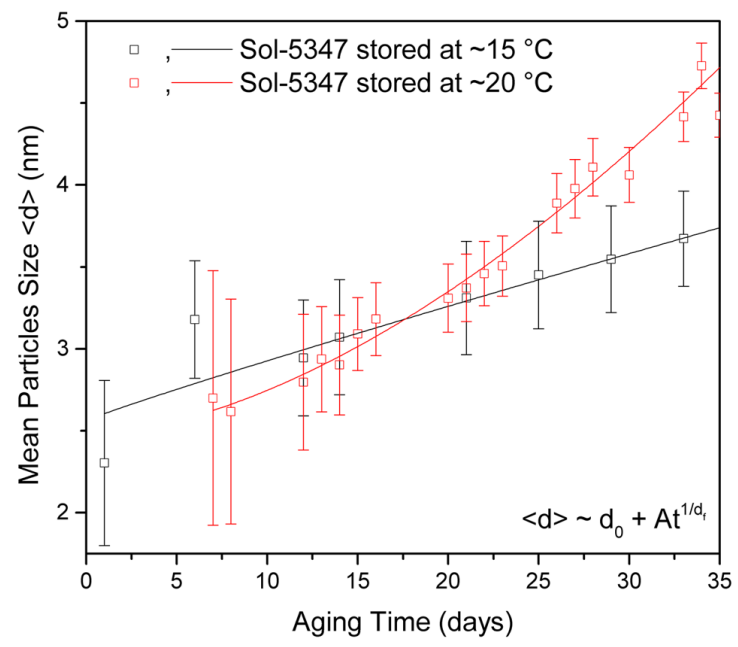

Figure 6. Evolution of the mean particles size as samples of a PZT 53/47 precursor aged under two different ambient conditions. Fittings are based on a DLCA aggregation mechanism.

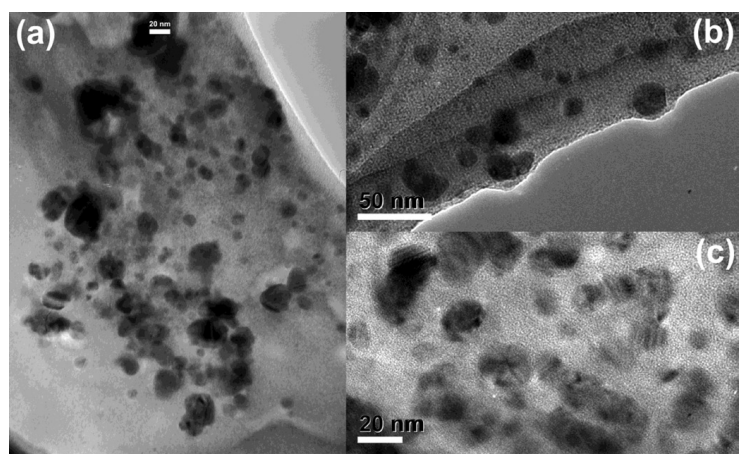

Figure 7. Representative (a) TEM and (b,c) HR-TEM images of our dried solutions for different magnifications. In this case, micrographs correspond to a Sol-9505 precursor. white squares, several zones uniformly oriented -denoted as A, B and C- were selected, Fourier-transformed and measured in order to determine the d-spacing and angles. The simulation of electron diffraction experiments by means of image processing techniques is also shown as well as the $\mathrm{d}$-spacing results for each of these regions. The superposition of those patterns may very well reproduce the raw Fourier transform of the original image that also appears as an inset in Figure 8b. According to the expected and previously described chemical environment, it is highly plausible to consider that these aggregates are formed by the $\mathrm{Zr}$ - and Ti- acetylacetonates compounds both quite structurally similar ${ }^{21,22}$ and with a noticeable chemical affinitythat may allow them to form this kind of spatial arrays when no solvent or dispersant is present. The precise determination of every chemical specie conforming those agglomerates is still a work in progress.

On the other hand, the second representative population of nanoparticles that showed up in our TEM analysis is shown in Figure 9.

In this case, a noticeable population of very small particles that, on average, do not tend to coalesce during solvent evaporation was observed. This kind of nanoparticles, showing a different spatial arrangement, could fairly consists on the highly dissociated and smaller $\mathrm{Pb}$ - acetate and/or methoxyethoxy complexes given that their interplanar distances, determined as it was described just above, could be associated to some of the already reported data ${ }^{17,23}$ for both complexes. Just as stated above, further work with the HR-TEM results is needed in order to univocally identify the nature of every compound present in our dried samples.

It must be worth noting that all of our microscopy results very well correlate with thecorresponding distribution curves already shown and discussed in this work for aged samples beyond their 'unimodal age'.

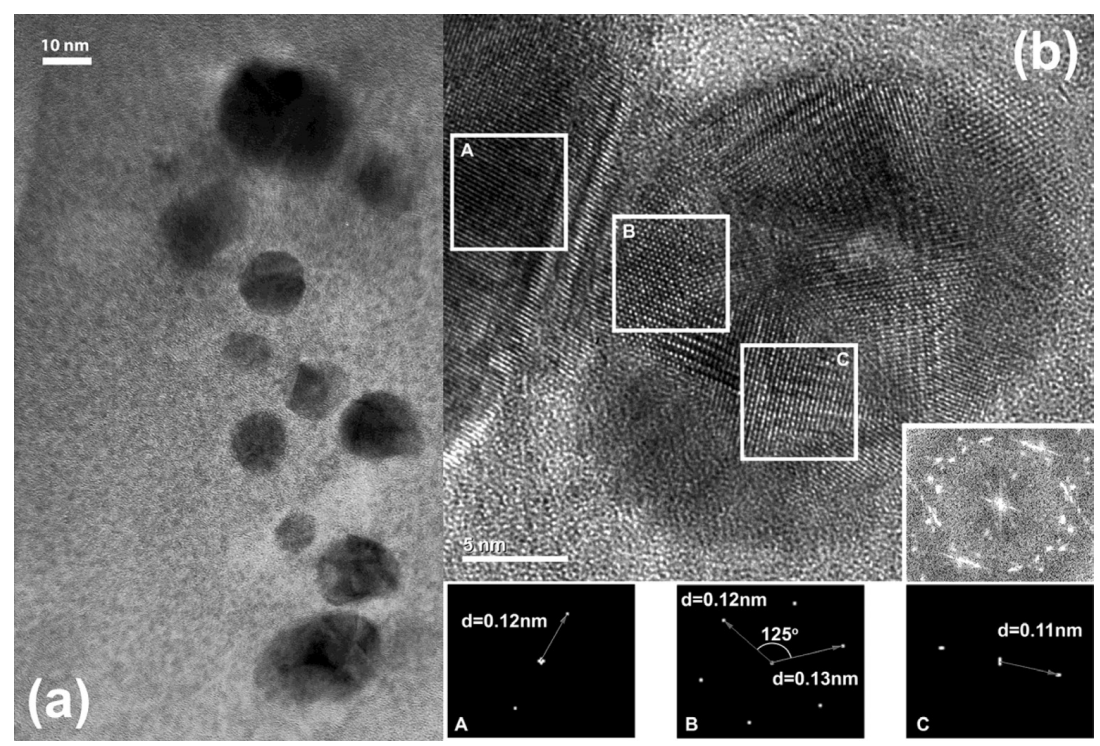

Figure 8. A typical (a) TEM and (b) HR-TEM view of the bigger aggregates of nanoparticles that were found in our dried samples. Inset in (b) represents the Fourier transform of (b) and regions A, B and C were chosen to analyze their own Fourier transforms which are correspondingly depicted below as well as the measured d-spacings and angles. In this case, micrographs correspond to a Sol-8020 precursor. 


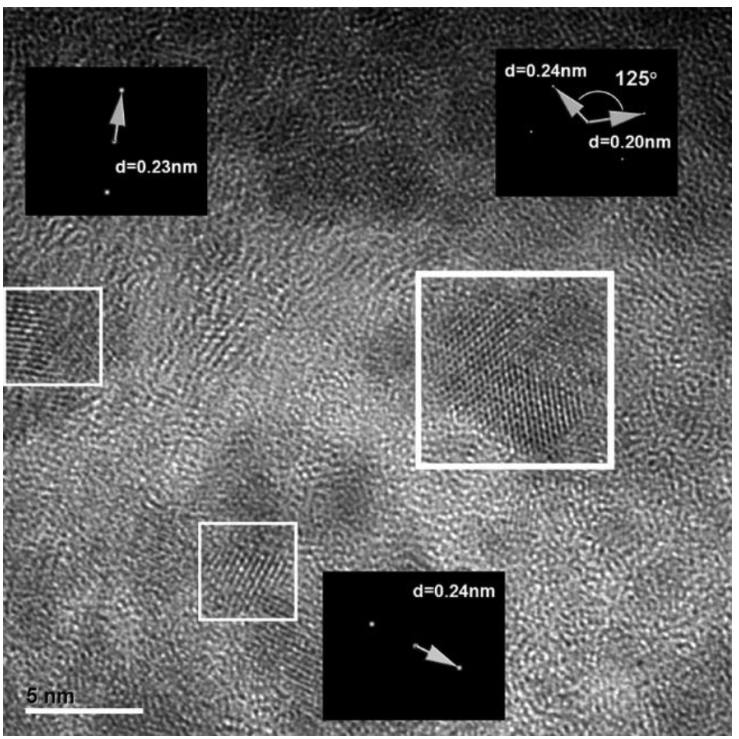

Figure 9. A typical HR-TEM view of the highly monodispersed and small nanoparticles obtained as our precursors dried. The insets show the determination of planar distances and angles by means of simulated electron diffraction experiments. In this case, micrographs correspond to a Sol-2575 precursor.

\section{Conclusions}

In this work we extended some of our previous results to show that, for our chosen chemical synthesis route, any PZT $(1-x) / x$ sol-gel based precursor could be highly stabilized with a remarkably tunable average particle size below $10 \mathrm{~nm}$

\section{References}

1. Zhuiykov S. Nanostructured semiconductor oxides for the next generation of electronics and functional devices: properties and applications. Woodhead Publishing; 2014. Structural and chemical modification of semiconductor nanocrystals; $p$. 50-94. Woodhead Publishing Series in Electronic and Optical Materials.

2. Athar T. Metal oxide nanopowder. In: Ahmed W and Jackson MJ, editors. Emerging nanotechnologies for manufacturing. Elsevier; 2009.

3. Hench LL and West JK. The sol-gel process. Chemical Reviews. 1990; 90(1):33-72. http://dx.doi.org/10.1021/cr00099a003.

4. Martin CR. Membrane-based synthesis of nanomaterials. Chemistry of Materials. 1996; 8(8):1739-1746. http://dx.doi. org/10.1021/cm960166s.

5. Martin CR. Template synthesis of electronically conductive polymer nanostructures. Accounts of Chemical Research. 1995; 28(2):61-68. http://dx.doi.org/10.1021/ar00050a002.

6. Wang F-P, Yu Y-J, Jiang Z-H and Zhao L-C. Synthesis of Pb1xEux(Zr0.52Ti0.48)O3 nanopowders by a modified sol-gel process using zirconium oxynitrate source. Materials Chemistry and Physics. 2003; 77(1):10-13. http://dx.doi.org/10.1016/ S0254-0584(01)00586-7.

7. Linardos S, Zhang Q and Alcock JR. Preparation of submicron PZT particles with the sol-gel technique. Journal of the European Ceramic Society. 2006; 26(1-2):117-123. http:// dx.doi.org/10.1016/j.jeurceramsoc.2004.10.007. and, specifically, corroborated the critical relevance of the stocking time to achieve this while trying to maximize the shelf-life of the specimens. Kinetically speaking, our systems undergo redissolution for several days before turning into a very stable, highly homogenized colloidal dispersion with an expected diffusion limited aggregation (DLCA) dominant mechanism.

By lookingback at our results, and simultaneously considering the several synthesis conditions that were enlisted in the 'Background' section, it looks feasible the selection of any "working point" in the PZT phase diagram to obtain similar results and this alone fact, tightly linked to our research or technological needs, might motivate similar studies and applications extended to other material systems of relevance.

\section{Acknowledgements}

We would like to sincerely acknowledge the invaluable help provided by Dr. J.M. Saniger-Blesa, Dr. R. ZanellaSpecia, Dr. P. Roquero-Tejeda and Dr. R.I. Puente-Lee from UNAM, Mexico and to Dr. V. Garibay-Febles from IMP, Mexico. It must be acknowledged the funding provided by PIFI (Programa Integral de Fortalecimiento Institucional) to the Project No. P/PIFI-2011-14MSU0010Z-16 for the acquisition of the Malvern Zetasizer Nano ZS90 equipment used in this research and to Project PROMEP-NPTC No. UDG-PTC-1080. Dr. Santiago J.A. Figueroa, from the Brazilian Synchrotron Light Laboratory (LNLS) at Campinas, must also be acknowledged for his fruitful recommendations.

8. Piwoński I. Mesoscopic objects, porous layers and nanocomposites: possibilities of sol-gel chemistry. Applied Surface Science. 2009; 256(3):S86-S91. http://dx.doi.org/10.1016/j.apsusc.2009.06.039.

9. Feynman RP. There's plenty of room at the bottom: an invitation to open up a new field of physics. Engineering and Science. 1960; 23(5):22-36.

10. Sidik NAC, Mohammed HA, Alawi OA and Samion S. A review on preparation methods and challenges of nanofluids. ICHMT. 2014; 54:115-125.

11. Haddad Z, Abid C, Oztop HF and Mataoui A. A review on how the researchers prepare their nanofluids. International Journal of Thermal Sciences. 2014; 76:168-189. http://dx.doi. org/10.1016/j.ijthermalsci.2013.08.010.

12. Suárez-Gómez A, Saniger-Blesa JM and Calderón-Piñar F. A study on the stability of a PZT precursor solution based on the time evolution of mean particles size and $\mathrm{pH}$. Materials Chemistry and Physics. 2010; 123(1):304-308. http://dx.doi. org/10.1016/j.matchemphys.2010.04.017.

13. Suárez-Gómez A, Sato-Berrú R, Toscano RA, Saniger-Blesa $\mathrm{JM}$ and Calderón-Piñar F. On the synthesis and crystallization process of nanocrystalline PZT powders obtained by a hybrid sol-gel alkoxides route. Journal of Alloys and Compounds. 2008; 450(1-2):380-386. http://dx.doi.org/10.1016/j.jallcom.2006.10.143.

14. Suárez-Gómez A, Saniger-Blesa JM and Calderón-Piñar F. The effects of aging and concentration on some interesting Sol-gel parameters: a feasibility study for PZT nanoparticles insertion on in-house prepared PAA matrices via electrophoresis. Journal 
of Electroceramics. 2009; 22(1-3):136-144. http://dx.doi. org/10.1007/s10832-007-9367-0.

15. Jaffe B, Cook WR and Jaffe HL. Piezoelectric ceramics. London: Academic Press; 1971.

16. Suárez-Gómez A, Sanchez-Tizapa M, Castañeda-Valderrama R and Carreón-Álvarez MA. Influence of chelation ratio of metal alkoxides on aging of PZT 53/47 sol-gel-based precursors. Bulletin of Materials Science. 2014; 37(5):1123-1129. http:// dx.doi.org/10.1007/s12034-014-0052-z.

17. Kolb U, Gutwerk D, Beudert R and Bertagnolli H. An IR- and EXAFS-study of the precursor system lead(II) acetate trihydrate, dissolved in methanol and 2-methoxyethanol. Journal of NonCrystalline Solids. 1997; 217(2-3):162-166. http://dx.doi. org/10.1016/S0022-3093(97)00153-1.

18. Lin MY, Lindsay HM, Weitz DA, Klein R, Ball RC and Meakin P. Universal diffusion-limited colloid aggregation. Journal of Physics Condensed Matter. 1990; 2(13):3093-3113. http:// dx.doi.org/10.1088/0953-8984/2/13/019.
19. Lin MY, Lindsay HM, Weitz DA, Ball RC, Klein R and Meakin P. Universal reaction-limited colloid aggregation. Physical Review A. 1990; 41(4):2005-2020. http://dx.doi.org/10.1103/ PhysRevA.41.2005. PMid:9903311.

20. Kolmogorov AN. Sulla determinazione empirica di una legge di distribuzione. Giornale dell'Istituto Italiano degli Attuari. 1933; 4:83-91.

21. Silverton JV and Hoard JL. Stereochemistry of discrete eight-coördination. II. The crystal and molecular structure of zirconium(iv) acetylacetonate. Inorganic Chemistry. 1963; 2(2):243-249. http://dx.doi.org/10.1021/ic50006a002.

22. Hoard JL and Silverton JV. Stereochemistry of Discrete EightCoördination. I. Basic Analysis. Inorganic Chemistry. 1963; 2(2):235-242. http://dx.doi.org/10.1021/ic50006a001.

23. Bryant RG, Chacko VP and Etter MC. Carbon-13 CP/MAS NMR and crystallographic investigations of the structure and solid-state transformations of lead(II) acetate trihydrate. Inorganic Chemistry. 1984; 23(22):3580-3584. http://dx.doi. org/10.1021/ic00190a029. 